\title{
The Fanaroff-Riley transition and the optical luminosity of the host elliptical galaxy
}

\author{
Gopal-Krishna $^{1}$ and P. J. Wiita ${ }^{2}$ \\ 1 National Centre for Radio Astrophysics, Tata Institute of Fundamental Research, Pune University Campus, \\ Post Bag No. 3, Ganeshkhind, Pune 411 007, India \\ 2 Department of Astrophysical Sciences, Princeton University, Princeton, NJ 08544-1001, USA \\ e-mail: wiita@astro.princeton.edu \\ on leave from the Department of Physics \& Astronomy, Georgia State University, University Plaza, Atlanta, \\ GA 30303-3083, USA \\ e-mail: wiita@chara.gsu.edu
}

Received 14 November 2000 / Accepted 9 April 2001

\begin{abstract}
We show that a model for radio source dynamics we had earlier proposed can readily reproduce the relationship between the radio power division separating the two Fanaroff-Riley classes of extragalactic radio sources and the optical luminosity of the host galaxy, as found by Owen \& Ledlow (1994). In our scenario, when less powerful jets eventually slow down to the point that the advance of the working surface (i.e., hotspot) becomes subsonic with respect to the external gas, the jet's collimation is severely weakened. This criterion distinguishes the powerful and well collimated FR II sources from the weaker sources producing the less collimated FR I type morphologies.
\end{abstract}

Key words. galaxies: active - galaxies: elliptical and lenticular, cD - galaxies: jets - galaxies: nuclei - radio continuum: galaxies

\section{Introduction}

A landmark in the study of extragalactic radio sources was the demonstration by Fanaroff \& Riley (1974) of the existence of a relatively sharp morphological transition at a radio luminosity of $P_{R}^{*}$ corresponding to $P_{178 \mathrm{MHz}} \simeq$ $2.5 \times 10^{26} h_{50}^{-2} \mathrm{~W} \mathrm{~Hz}^{-1}$. The great majority of sources below this luminosity (FR I type) are characterized by having diffuse radio lobes, with their brightest regions within the inner half of the radio source, and so can be called edgedimmed. On the contrary, more powerful sources are usually straighter, exhibit edge-brightened (FR II) morphology, and typically contain hotspots near the outer edges of their radio lobes.

More recently it was realized that the critical radio luminosity separating the FRI and FR II actually increases with the optical luminosity of the host elliptical galaxy, so that $P_{R}^{*} \propto L_{\mathrm{opt}}^{1.65}$ (as measured from Fig. 1 of Ledlow \& Owen 1996); also see Owen \& White (1991); Owen \& Ledlow (1994) for earlier indications of this effect. Although many detailed differences between the host galaxies of the FR I and FR II sources have been discovered (e.g., Baum et al. 1995; Zirbel 1997; for a recent

Send offprint requests to: Gopal-Krishna,

e-mail: krishna@ncra.tifr.res.in summary, see Gopal-Krishna \& Wiita 2000), it is fair to state that a key fact is that the more luminous the host galaxy is, the more powerful the radio source must be in order to attain the FR II morphological classification.

The most widely known explanation for the difference between the FR I and FR II sources is that, while the jets in both cases start out moving at very high (relativistic) speeds, those in FR II sources remain that way out to multi-kpc distances, while those in the FR I's decelerate to much lower speeds within a few kpc of the core (e.g., Begelman 1982; Bicknell 1984; De Young 1993; Komissarov 1994; Laing 1993, 1996; Laing et al. 1999). Detailed models for decelerating relativistic jets were developed by Bicknell $(1994,1995)$. The core of his argument is that relativistic jets, once they come into pressure equilibrium with the external interstellar medium (ISM) or intracluster medium (ICM), will become strongly KelvinHelmholtz unstable and then entrain substantial amounts of cold ambient material. Bicknell (1995) showed that this instability typically set in as the internal jet Mach number approaches $\sim 2$, corresponding to internal bulk velocities having dropped to about $0.6 c$.

Of course, many other explanations for the FR I/FR II dichotomy have been proposed (e.g., Baum et al. 1995; Reynolds et al. 1996a, 1996b; Meier 1999; 
Valtonen \& Heinämäki 2000; see the recent discussion by Gopal-Krishna \& Wiita 2000). Some of these proposals involve fundamental differences in the nature of the jets (electron/positron vs. electron/proton plasma) or of the central engine (black hole spin, type of accretion disk). The viability of any member of this class of explanations is challenged by the existence of HYbrid MOrphology Radio Sources, or HYMORS, which have a clear FR I morphology on one side of the host galaxy, but a distinct FR II morphology on the other side (Gopal-Krishna \& Wiita (2000, 2001).

An alternative approach posits that FR I and FR II sources differ primarily in the importance of the beam thrust relative to the basic parameters of the ambient medium (Gopal-Krishna \& Wiita 1988 (GKW88); Gopal-Krishna 1991 (GK91); Gopal-Krishna et al. 1996 (GKWH96); Blandford 1996). In this version of the deceleration scenario, the emphasis is on the slowing of the advance of the hotspot, or working surface, at the end of the jet, rather than on the slowing of the bulk flow within the jet (e.g., Bicknell 1995). When the hotspot's advance becomes transonic relative to the ambient medium, its Mach disk weakens considerably due to the fall in ram pressure, and the jet becomes decollimated; this soon leads to an FR I morphology. Further expansion of such jets beyond this point is expected to be in the form of a plume, as discussed in GKW88, where it was also shown that for reasonable values of jet and galaxy parameters (density, core radius and temperature of the ISM) such a jet flaring can occur within several kpc of the core for jet powers below about $10^{43} \mathrm{erg} \mathrm{s}^{-1}$, consistent with the observations of the FR I/FR II division (GKW88; GK91; GKWH96). Also, in this picture, the concomitant dimunition/cessation of the "backflow" of the beam plasma would cause a depletion of the protective sheath of beam plasma around the jet, facilitating entrainment of the ISM material into the jet flow.

Bicknell (1995) expanded his model to account for the Owen-Ledlow transition in the $P_{R}-L_{\text {opt }}$ plane. A key ingredient in his scenario involved connecting the jet dynamics to empirically established relations between the optical magnitude of the galaxy on the one hand, and the soft $\mathrm{X}$-ray luminosity, core radius, and central velocity dispersion of elliptical galaxies on the other hand. In the present study, we follow Bicknell and adopt these same empirical relationships. Bicknell then ties the galactic parameters to those of the jet by demanding that the establishment of a pressure balance between the jet and the external medium corresponds closely to the location of the jet's internal transonic transition. By doing so, he was able to derive a formula connecting beam power to the optical luminosity of the host galaxy. In this paper we follow a different approach in this latter stage.

Additional assumptions on the efficiency of the conversion of the jet's energy into total radio luminosity $(>1-2 \%)$, and thence into monochromatic radio emission, allowed Bicknell (1995) to obtain a fairly good fit to the slope of the Owen-Ledlow division, finding that
$P_{R}^{*} \propto L_{\mathrm{opt}}^{2.1}$, as well as getting a decent fit to the intercept; however, there were quite a few poorly constrained parameters in his model. Nevertheless, Bicknell (1995) argued that the the slope of the relation was rather insensitive to the likely uncertainties in parameters. He further argued that the intercept would tend to be driven towards better agreement with the data for plausible variations in those parameters, which included: the ratio of the jet's lifetime to the synchrotron cooling time of the highest energy electrons; the ratio of the upper and lower cutoff energies for the electron distribution; the ratio of jet radius to its length at the transition distance; the ratio of the product of the jet pressure and square of its radius evaluated at the transition radius to that of the external medium evaluated at its core radius; the bulk velocity of the jet at the transition point; the low frequency radio spectral index; and the value of an integral (discussed below) which depends on an upper cut-off radius for the X-ray emitting halo of the host galaxy.

The new magnetic switch model (Meier 1999) fundamentally distinguishes FR I from FR II sources through the different speeds of rotation of the magnetic field lines of their central engines, which are tied to the different spin rates of their supermassive black holes. While this scenario can also produce a slope for the $P_{R}^{*}-L_{\mathrm{opt}}$ dividing line close to that found by Ledlow \& Owen (1996) (Meier 1999) we recall that it is hard to reconcile this scheme with the existence of HYMORS (Gopal-Krishna \& Wiita 2000, 2001). Venturing beyond the standard nuclear jet paradigm, gravitational slingshot models can in principle be compatible with the Owen-Ledlow relation (Valtonen \& Heinämäki 2000), and could also account for HYMORS (Gopal-Krishna \& Wiita 2000).

\section{The model}

In an earlier study involving "weak headed quasars", where prominent one-sided jets are not seen to terminate in a conspicuous hotspot, we have argued that dissipation of jet power, as suggested by Swarup et al. (1982) and Saikia et al. (1983), may actually not be responsible for the lack of terminal hotspots (GKWH96). Taking a clue from the fact that no two-sided jets are seen in these sources, we argued that the lack of a hotspot could be best explained through the onset of a jet's decollimation when the hotspot's (or, nearly equivalently, the bow shock's) velocity becomes transonic relative to the external medium (GKWH96). This successful explanation of this class of radio sources motivates us to seek an explanation for the Owen-Ledlow relation in terms of a similar scenario. This model makes no assumptions about the relativistic nature of the bulk velocity of the internal jet fluid, which may be gradually decelerating as the head of the jet advances.

Our study makes use of the same empirical relations between the elliptical's blue magnitude, $M_{B}$, and its soft X-ray emission, $L_{\mathrm{X}}$ (Donnelly et al. 1990), stellar velocity dispersion, $\sigma$ (Terlevich et al. 1981 the Faber-Jackson relation), and X-ray core radius, a 
(Kormendy 1987), as employed by Bicknell (1995), assuming $H_{0}=75 \mathrm{~km} \mathrm{~s}^{-1} \mathrm{Mpc}^{-1}$ :

$\log L_{\mathrm{X}}=22.3-0.872 M_{B}$,

$\log \sigma=5.412-0.0959 M_{B}$,

$\log a=11.7-0.436 M_{B}$.

However, we adopt the initially conical jet model based on our earlier work (Gopal-Krishna \& Wiita 1987), which was generalized in Gopal-Krishna et al. (1989, GKWS89) to allow for bulk relativistic jet flow whereby the hotspot motion can also be relativistic. Ram pressure balance, under these conditions, gives the hotspot velocity, $v$, as a function of distance, $D$, from the central engine, as (GKWS89):

$$
v(D)=\frac{X c\left[1+(D / a)^{2}\right]^{\delta / 2}}{D+X\left[1+(D / a)^{2}\right]^{\delta / 2}} .
$$

Here $X=\left(4 L_{\mathrm{b}} / \pi c^{3} \theta^{2} n_{o} m_{p} \mu\right)^{1 / 2}$, where $L_{\mathrm{b}}$ is the jet (beam) power, $\theta$ is the jet's effective opening angle, $m_{p}$ and $\mu$ have their usual definitions, and $n_{0}$ is the central density of the ISM, which is taken to fall off as (e.g., Forman et al. 1985; Canizares et al. 1987; Conway 2001)

$n(D)=\frac{n_{0}}{\left[1+(D / a)^{2}\right]^{\delta}}$.

For our calculations we choose $\theta=0.1 \mathrm{rad}$, which is certainly justified as appropriate for the inner jet regions, particularly since it is now recognized that the jet's thrust acts on a larger area than the instantaneous hot spot (e.g., Scheuer's 1982 "dentist-drill", as supported by many three-dimensional numerical simulations: Norman 1996; Clarke 1996; Kaiser \& Alexander 1997; Hooda \& Wiita 1998). Following Bicknell (1995), we further assume that the X-ray emitting ISM gas temperature is tied to the central stellar velocity dispersion, $\sigma$, via, $k T=2.2 \mu m_{p} \sigma^{2} / \delta$, which is Fall's (1987) relation between circular velocity and $\sigma$. Here $\delta$ is roughly 0.75 , as inferred from soft X-ray images of nearby ellipticals (e.g., Forman et al. 1985; Canizares et al. 1987; Sarazin 1986), as well as dynamical models of radio sources (e.g., Gopal-Krishna \& Wiita 1991; Conway 2001).

Studies of many sub-classes of young radio sources, including Compact Symmetric Objects (e.g., Owsianik \& Conway 1998; Conway 2001), Gigahertz Peaked Sources (Carvalho 1999) and Compact Steep Spectrum radio sources (Gopal-Krishna \& Wiita 1991; Jeyakumar et al. 2001) have indicated that the mean density of the gas interacting with the radio lobes in the inner $\sim 1 \mathrm{kpc}$ or so is typically a few atoms $\mathrm{cm}^{-3}$, or several times that estimated from X-ray emission. This evidence in favor of a significant contribution of cooler gas to the ambient medium (at least in the inner portions of elliptical galaxies) is further supported by studies of the linear-size distribution of these radio sources (O'Dea \& Baum 1997). We shall parameterize the relation between the confining central density, $n_{0}$, used in Eq. (5) and that derived from X-ray measurements, $n_{\mathrm{X}}$ as follows: $n_{0}=\kappa n_{\mathrm{X}}$, with $\kappa \geq 1$; the fiducial value we use below is $\kappa=3$.

The radio data cited above as well as the relation between $M_{B}$ and the X-ray core radius (Eq. (3)) lead to values of of $a \sim 1 \mathrm{kpc}$. Therefore the ISM density begins to approach the large scale behavior, and thus declines quite rapidly, at radial distances beyond a few kpc. Hence, the most likely regime for the jets' decollimation due to the hot spots having slowed to subsonic speeds lies within roughly $10 \mathrm{kpc}$ of the core. If the hot spot manages to retain supersonic speed out to such radial distances, then in most cases the jet is likely to propagate ahead down the rapidly declining ISM density, preserving its FR II character. In some cases another possibility for jet flaring can arise farther out when the jet crosses the pressure-matched interface between the ISM and the intergalactic medium (GKW88; Wiita et al. 1990; Wiita \& Norman 1992; Hooda et al. 1994; Hooda \& Wiita 1996, 1998; Zhang et al. 1999). Relatively few sources will, however, make a transition to FR I morphology upon crossing this interface since a significant degree of beam collimation appears to occur by such radial distances within the extended halos of most galaxies (Blundell et al. 2001; Jeyakumar \& Saikia 2000). For such very extended sources, our model is clearly oversimplified, as the evolution appears to break from selfsimilarity and the decrease in $P_{R}$ with time (even for constant $L_{\mathrm{b}}$ ) cannot be ignored (e.g., Blundell \& Rawlings 2000).

Other types of sources that may not be readily accommodated in our simple picture are, for instance, cases where a narrow jet is seen to terminate in a bright knot far away (say $>50 \mathrm{kpc}$ ) from the nucleus, and flares only beyond that point (e.g., 3C 130, Hardcastle 1998). Of course, higher values of $D^{*}$ are expected for sources for which the beam's power and opening angle lie near the upper and lower ends of their respective ranges. Also, the possibility of a bright knot and jet expansion occurring when the jet interacts with irregularities in the ambient medium must be considered (e.g., Wang et al. 2000). In this context it is especially interesting to recall that recent radio observations of the nearest radio galaxy, Centaurus A, have provided clear evidence that a radio jet can even emerge recollimated after flaring upon hitting a gas cloud (Morganti et al. 1999). These Australian Telescope Compact Array observations have revealed that the flaring of the northern jet of Cen A, a few kiloparsecs away from from the nucleus, gives rise to the inner radio "lobe", out of which a narrow jet is seen to emerge and propagate further out for another few kiloparsecs before flaring and fading away in the fashion of typical FR I jets. Morganti et al. (1999) have interpreted this striking "leaky bulb" type morphology as being a manifestation of the de Laval nozzle model proposed by Blandford \& Rees (1974).

In our analytical formulation, we evaluate the critical beam power, $L_{\mathrm{b}}^{*}$, for which the hot-spot deceleration to subsonic velocities occurs at a fiducial distances, $D^{*}$, of 3 and $10 \mathrm{kpc}$ from the core, for a range of absolute 
magnitudes of the host galaxy between $M_{B}=-19$ and -23.5. The $10 \mathrm{kpc}$ value is a typical distance at which jets flare in a sample of radio galaxies (O'Donoghue et al. 1993). A recent study of a sample of 38 FR I sources by Laing et al. (1999) gives a mean projected value of $\sim 3.5 \mathrm{kpc}$ for the radial distance of the point where the kiloparsec scale jet first becomes visible, after an initial emission gap (using our value of $H_{0}$ ). Our main results are not very sensitive to the choice of $D^{*}$, at least until the very most luminous galaxies are considered, and the predicted slope of the separation between FR I and FR II sources that we derive below is not strongly dependent upon it.

We now write the relevant expressions as follows:

$X=C_{2} n_{0}^{-1 / 2} L_{\mathrm{b}}^{1 / 2}$

where $C_{2}=\left(4 / \pi c^{3} \theta^{2} m_{\mathrm{p}} \mu\right)^{1 / 2}=2.14 \times 10^{-3}$ for $\mu=0.620$. We also require, $C_{1}=4 \pi(0.909) \Lambda(T) I_{\mathrm{X}}$, where the cooling function, $\Lambda(T)$ is a rather weak function of the temperature; assuming $T=10^{7} \mathrm{~K}, \Lambda(T) \simeq 1.17 \times 10^{-23}$ (Bicknell $1995)$, and the integral over the gas density distribution gives $I_{\mathrm{X}} \simeq 5.0$ (for $\delta=0.75$ ), a value we adopt, following Bicknell (1995). With these choices, $C_{1}=6.68 \times 10^{-22}$.

Equations (1-3) and (6) can now be combined to yield:

$\log L_{\mathrm{X}}=\log C_{1}+2 \log n_{0}+3 \log a$,

$\log n_{\mathrm{X}}=\log n_{0}-\log \kappa=4.188+0.218 M_{B}$,

$$
\begin{aligned}
\log X= & -0.109 M_{B}+\log C_{2}+0.25 \log C_{1}+3.20 \\
& +0.5 \log L_{\mathrm{b}} .
\end{aligned}
$$

We next impose the transonic condition on the LHS of Eq. (4), via

$v(D)=c_{\mathrm{s}}=[\gamma 2.2 / \delta]^{1 / 2} \sigma$.

Taking the ratio of specific heats for the combined plasma, $\gamma=5 / 3$, and using $\delta=0.75$, this becomes

$\log v(D)=0.345+\log \sigma=-4.720-0.0959 M_{B}$.

Equating this to the RHS of (4), treated as a function of $X$, gives,

$\operatorname{dex}\left(-4.720-0.0959 M_{B}\right)=\frac{X}{D /\left[1+(D / a)^{2}\right]^{\delta / 2}+X}$,

as the relation that must be solved for $L_{\mathrm{b}}^{*}$ in terms of $M_{B}$.

\section{Results and conclusions}

The values of $L_{\mathrm{b}}^{*}, a$, and $n_{0}$ can be found for specified values of $M_{B}$ and $D^{*}$ by solving Eqs. (3), (8), (9) and (12). The results are given in Table 1 , along with, $\beta$, the local slope of the $\log L_{\mathrm{b}}^{*}-\log L_{\mathrm{opt}}$ relation. For the choice of $D^{*}=10 \mathrm{kpc}$, these slopes, $\beta$, vary little for $M_{B}>-23.5$, and correspond closely to a relation where $L_{\mathrm{b}}^{*} \propto L_{\mathrm{opt}}^{1.6}$; for $D^{*}=3 \mathrm{kpc}, \beta$ is only slightly less steep. For the most luminous galaxies, the value of $\beta$ from the model flattens somewhat.
Table 1. Galaxy and jet parameters.

\begin{tabular}{ccccccc}
\hline$M_{B}$ & $\begin{array}{c}a \\
(\mathrm{kpc})\end{array}$ & $\begin{array}{c}n_{0} \\
\left(\mathrm{~cm}^{-3}\right)\end{array}$ & $\begin{array}{c}L_{\mathrm{b}, D^{*}=10} \\
\left(\mathrm{erg} \mathrm{s}^{-1}\right)\end{array}$ & $\beta_{D^{*}=10}$ & $\begin{array}{c}L_{\mathrm{b}, D^{*}=3} \\
\left(\mathrm{erg} \mathrm{s}^{-1}\right)\end{array}$ & $\beta_{D^{*}=3}$ \\
\hline-19.5 & 0.052 & 2.82 & $4.35(41)$ & 1.74 & $2.42(41)$ & 1.80 \\
-20.0 & 0.085 & 2.02 & $8.28(41)$ & 1.40 & $4.53(41)$ & 1.36 \\
-20.5 & 0.141 & 1.57 & $1.71(42)$ & 1.57 & $7.67(41)$ & 1.58 \\
-21.0 & 0.233 & 1.22 & $3.57(42)$ & 1.60 & $1.92(42)$ & 1.56 \\
-21.5 & 0.384 & 0.951 & $7.23(42)$ & 1.56 & $3.93(42)$ & 1.55 \\
-22.0 & 0.635 & 0.741 & $1.49(43)$ & 1.58 & $7.92(42)$ & 1.52 \\
-22.5 & 1.05 & 0.576 & $3.06(43)$ & 1.55 & $1.56(43)$ & 1.47 \\
-23.0 & 1.73 & 0.447 & $6.21(43)$ & 1.55 & $2.80(43)$ & 1.27 \\
-23.5 & 2.86 & 0.348 & $1.23(44)$ & 1.47 & $4.11(43)$ & 0.99 \\
\hline
\end{tabular}

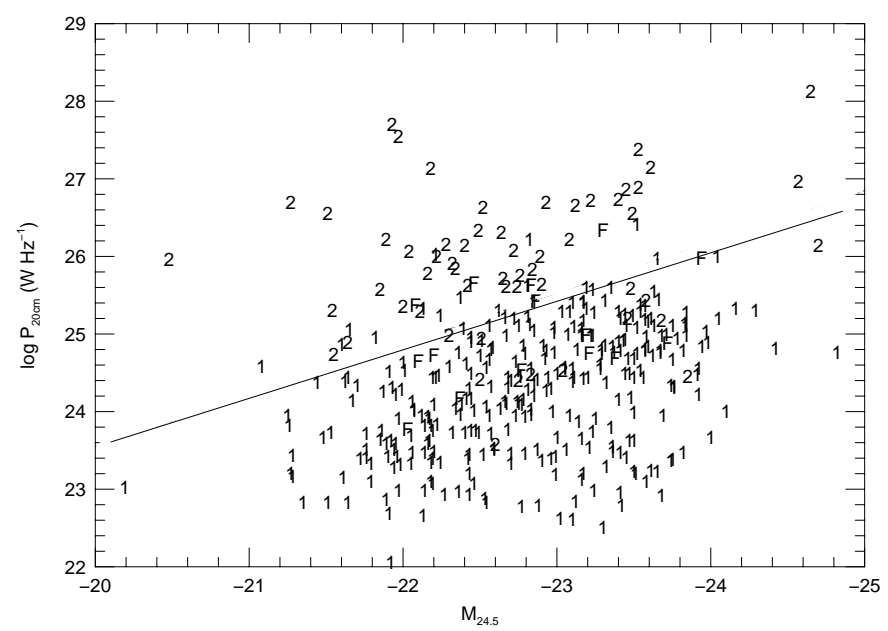

Fig. 1. Radio flux plotted against host galaxy magnitude, with the numerals indicating FR class; data taken from Ledlow et al. (2000). The superimposed curve is the division between FR I and FR II sources arising from our model, taking $D^{*}=10 \mathrm{kpc}$ and a constant fraction of total beam power converted into synchrotron emission of $\epsilon=0.09$.

In the absence of a definitive approach to compute the efficiency, $\epsilon$, of conversion of the beam power into synchrotron radiation, we make the commonly adopted first order approximation that the monochromatic radio luminosity is a constant fraction of the beam power (at least near the transition luminosity). Then the local slope remains the same, with $P_{R, 1.4 \mathrm{GHz}}^{*} \widetilde{\sim} L_{\text {opt }}^{1.56}$, and the key features of the Owen-Ledlow diagram are satisfactorily explained, with a very good fit to the slope retained. The intercept also fitted well, provided the product $\epsilon \kappa \simeq 0.27$ for $D^{*}=10 \mathrm{kpc}$. As shown in Fig. 1, this is in excellent agreement with the bifurcation between FR I and FR II radio sources, and nominally provides even a better fit than does Bicknell's (1995) model, which yields a power-law index above 2.0. We use a mean color index for elliptical galaxies $(B-R)=1.15$ taken from a CCD photometric study of cluster galaxies (Jørgensen et al. 1992) to convert from the absolute blue magnitudes used in Eqs. (1-3) to the red magnitudes plotted by Ledlow et al. (2001); the dependence of color index on optical magnitude is small over this range and we have not attempted to correct for it 
(Jørgensen et al. 1992; Kodama et al. 1998; Peletier et al. 1990).

We note that earlier estimates based on the application of beam models for FR II sources are in reasonable agreement with such values of $\epsilon \approx 0.1$ (Gopal-Krishna \& Saripalli 1984; Dreher 1984; Saripalli \& Gopal-Krishna 1985), particularly if a "ready to radiate" electronpositron jet composition is assumed; and evidence for such a composition is growing (e.g., Reynolds et al. 1996a; Kaiser \& Alexander 1997; Kaiser et al. 1997; Hirotani et al. 1999). Given that our analysis involves a fairly large set of empirical relations for elliptical galaxies, the agreement of our prediction with the observational data is quite encouraging (Fig. 1).

It is perhaps worth remarking on the consistency of our model with the recent Very Long Baseline Interferometry measurements for the expansion of very small and young Compact Symmetric Object (CSO) radio sources (e.g., Owsianik \& Conway 1998 (OC)). For the CSO 0710+439, the best values obtained by OC are $V \simeq 0.13 h^{-1} c$ at a distance $D \simeq 25 h^{-1}$ pc $(\ll a)$, for $n_{0} \simeq 2 \mathrm{~cm}^{-3}$, and $L_{\mathrm{b}} \simeq 5 \times 10^{44} \mathrm{erg} \mathrm{s}^{-1}$. Inserting the last three values into

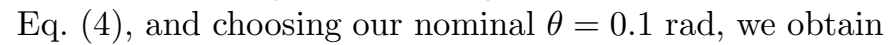
$V=0.18 h^{-1} c$, in quite reasonable agreement; a somewhat higher value for $\theta$ might be inferred from the maps in OC, thereby reducing our computed velocity and improving the agreement. Less well determined values of expansion speeds obtained by other groups for 3 other CSO radio sources quoted by OC range from $0.07-0.13 h^{-1} c$ and would easily be accommodated by more typical values of $L_{\mathrm{b}}$. The derived range, $0.08<\epsilon<0.31$, for $0710+439$ (OC) is also in good agreement with our model.

The forgoing analysis is admittedly oversimplified; for instance, we have considered a fixed opening angle for beams of all powers, and have also assumed specific values of $\delta$ and $\kappa$, all of which are certain to vary somewhat from source to source. On the other hand, the division between FR I and FR II sources is not perfect by any means, and such a spread in properties would allow for the small number of sources found on the "wrong" side of the dividing line in Fig. 1. Also, we have ignored any variation in the jet disruption length with the galaxy's optical luminosity by typically choosing $D^{*}=10 \mathrm{kpc}$; however, such a variation is quite plausible, and therefore we have also provided results for $D^{*}=3 \mathrm{kpc}$ in the last two columns in Table 1.

To obtain essentially as good a fit with $D^{*}=3 \mathrm{kpc}$, one would have to raise $\epsilon$ to 0.16 if $\kappa=3$ instead of $\epsilon=0.09$ for $D^{*}=10 \mathrm{kpc}$; of course both values could drop if $\kappa>3$ is considered. As a first step towards a more realistic model, one could consider a distribution of $2-10 \mathrm{kpc}$ for $D^{*}$ over the range in the hosts' optical magnitudes, taking smaller $D^{*}$ for lower $L_{\mathrm{opt}}$. Then the predicted slope of the $P_{R}^{*}-L_{\text {opt }}$ relation clearly would be slightly steeper (cf. Cols. 4 and 6 of Table 1), further improving the accord with the data presented by Ledlow \& Owen (1996) and recently updated by Ledlow et al. (2001). We also note that the exact results quoted assume a specific spectral index $(\alpha=1.0)$ and upper and lower cut-offs to the radio band $(10 \mathrm{MHz}$ and $100 \mathrm{GHz}$ ) respectively, in relating the monochromatic radio power to the beam power. Assuming a flatter overall spectrum, say $\alpha=0.7$, would not affect the slope of the relation but would demand that the product $\epsilon \kappa$ rise to $\simeq 0.52$ (for $D^{*}=10 \mathrm{kpc}$ ) to normalize it. Despite these uncertainties, it is probably fair to note that overall our variant model employs several fewer parameters than does Bicknell's (1995), although in connecting optical and X-ray properties we have closely followed his approach.

The available very detailed maps of a few kiloparsecscale FR I jets provide evidence for the existence of a slower moving sheath of synchrotron plasma surrounding a relativistic spine (e.g., Laing 1993, 1996; Laing et al. 1999). In Bicknell's picture this sheath arises from turbulent mixing of the ambient plasma after the jet's Mach number becomes low enough to allow an exponential growth of the planar Kelvin-Helmholtz instability. In our picture, the entrainment process is accelerated once the hotspot's motion has become subsonic, as this results in the cessation of the "backflow" of the jet plasma; this leads to the diminution of the protective cocoon around the jet core which had hitherto separated the latter from the ISM material. We also recall that the impingement of the "backflow" onto the jet engenders some of the reconfinement shocks (e.g. Norman et al. 1982; Hooda \& Wiita 1996, 1998) so that the depletion of the shrouding cocoon plasma reduces their effects and therefore contributes to the jet's decollimation. Thus, in our picture, the dramatic slowing down of the beam flow can be thought of as an eventual outcome of the decollimation of the beam's head (leading to FR I structure), instead of being its principal cause.

Acknowledgements. We thank Michael Ledlow and Frazer Owen for providing the data used in Fig. 1 and B. Premkumar for assistance in producing that figure. GK appreciates hospitality at Princeton University while much of this work was carried out. PJW acknowledges support from CST funds at Princeton and RPE funds at Georgia State University.

\section{References}

Baum, S. A., Zirbel, E. L., \& O'Dea, C. P. 1995, ApJ, 451, 88 Begelman, M. C. 1982, in IAU Symp. 97, Extragalactic Radio Sources, ed. D. S. Heeschen, \& C. M. Wade (Kluwer, Dordrecht), 223

Bicknell, G. V. 1984, ApJ, 286, 68

Bicknell, G. V. 1994, ApJ, 422, 542

Bicknell, G. V. 1995, ApJS, 101, 29

Blandford, R. D. 1996, in Cygnus A - Study of a Radio Galaxy, ed. C. Carilli, \& D. Harris (Cambridge Univ. Press, Cambridge), 264

Blandford, R. D., \& Rees, M. J. 1974, MNRAS, 169, 395

Blundell, K. M., \& Rawlings, S. 2000, ApJ, 119, 1111

Blundell, K. M., Rawlings, S., Willott, C. J., Kassim, N. E., \& Perley, R. A. 2001, New Astron. Rev., Proc. STScI Workshop on Lifecycles of Radio Galaxies, in press

Canizares, C. R., Fabbiano, G., \& Trinchieri, G. 1987, ApJ, 312,503

Carvalho, J. C. 1998, A\&A, 329, 845 
Clarke, D. A. 1996, in Energy Transport in Radio Galaxies and Quasars, ed. P. Hardee, A. H. Bridle, \& J. Zensus (ASP, San Francisco), ASP Conf. Ser., 100, 311

Conway, J. 2001, New Astron. Rev., Proc. STScI Workshop on Lifecycles of Radio Galaxies, in press

De Young, D. S. 1993, ApJ, 405, L13

Donnelly, R. H., Faber, S. M., \& O'Connell, R. M. 1990, ApJ, 354,52

Dreher, J. W. 1984, in Physics of Energy Transport in Extragalactic Radio Sources, ed. A. H. Bridle, \& J. A. Eilek (NRAO, Green Bank), 109

Fall, S. M. 1987, in Nearly Normal Galaxies from the Planck Time to the Present, ed. S. M. Faber (Springer Verlag, New York), 326

Fanaroff, B. L., \& Riley, J. M. 1974, MNRAS, 167, 31P

Forman, W., Jones, C., \& Tucker, W. 1985, ApJ, 293, 102

Gopal-Krishna 1991, A\&A, 248, 415 (GK91)

Gopal-Krishna, \& Saripalli, L. 1984, A\&A, 139, L19

Gopal-Krishna, \& Wiita, P. J. 1987, MNRAS, 226, 531

Gopal-Krishna, \& Wiita, P. J. 1988, Nature, 333, 49 (GKW88)

Gopal-Krishna, \& Wiita, P. J. 1991, ApJ, 373, 325

Gopal-Krishna, \& Wiita, P. J. 2000, A\&A, 363, 507

Gopal-Krishna, \& Wiita, P. J. 2001, New Astron. Rev., Proc. STScI Workshop on Lifecycles of Radio Galaxies, in press

Gopal-Krishna, Wiita, P. J., \& Hooda, J. S. 1996, A\&A, 316, L13 (GKWH96)

Gopal-Krishna, Wiita, P. J., \& Saripalli, L. 1989, MNRAS, 239, 173 (GKWS89)

Hardcastle, M. J. 1998, MNRAS, 298, 569

Heckman, T. M., et al. 1986, ApJ, 311, 526

Hirotani, K., Iguchi, S., Kimura, M., \& Wajima, K. 1999, PASJ, 51, 263

Hooda, J. S., Mangalam, A. P., \& Wiita, P. J. 1994, ApJ, 423, 116

Hooda, J. S., \& Wiita, P. J. 1996, ApJ, 470, 211

Hooda, J. S., \& Wiita, P. J. 1998, ApJ, 493, 81

Jeyakumar, S., \& Saikia, D. J. 2000, MNRAS, 311, 397

Jeyakumar, S., Wiita, P. J., Saikia, D. J., \& Hooda, J. S. 2001, submitted to ApJ

Jørgensen, I., Franx, M., \& Kjærgaard, P. 1992, A\&AS, 95, 489

Kaiser, C. R., \& Alexander, P. 1997, MNRAS, 286, 215

Kaiser, C. R., Dennett-Thorpe, J., \& Alexander, P. 1997, MNRAS, 292, 723

Kodama, T., Arimoto, N., Barger, A. J., \& Aragón-Salamanca, A. 1998, A\&A, 334, 99

Komissarov, S. S. 1994, MNRAS, 269, 394

Kormendy, J. 1987, in Structure and Dynamics of Elliptical Galaxies, ed. T. de Zeeuw (Reidel, Dordrecht), 78

Laing, R. A. 1993, in STScI Symp. 6: Astrophysical Jets, ed. D. Burgarella, M. Livio, \& C. O'Dea (Cambridge Univ. Press, Cambridge), 95
Laing, R. A. 1996, in Energy Transport in Radio Galaxies and Quasars, ed. P. Hardee, A. H. Bridle, \& J. Zensus (ASP, San Francisco), ASP Conf. Ser., 100, 241

Laing, R. A., Parma, P., de Ruiter, H. R., \& Fanti, R. 1999, MNRAS, 306, 513

Ledlow, M. J., \& Owen, F. N. 1996, AJ, 112, 9

Ledlow, M. J., Owen, F. N., \& Eilek, J. A. 2001, New Astron. Rev., Proc. STScI Workshop on Lifecycles of Radio Galaxies, in press

Meier, D. L. 1999, ApJ, 522, 753

Meier, D. L., Edgington, S., Godon, P., Payne, D. G., \& Lind, K. R. 1997, Nature, 388, 350

Morganti, R., Killeen, N. E. B., Ekers, R. D., \& Oosterloo, T. A. 1999, MNRAS, 307, 750

Norman, M. L. 1996, in Energy Transport in Radio Galaxies and Quasars, ed. P. Hardee, A. H. Bridle, \& J. Zensus (ASP, San Francisco), ASP Conf. Ser., 100, 319

Norman, M. L., Winkler, K.-H. A., Smarr, L., \& Smith, M. D. 1982, A\&A, 113, 285

O'Dea, C. P., \& Baum, S. A. 1997, AJ, 113, 148

O'Donoghue, A. A., Eilek, J. A., \& Jones, J. M. 1993, ApJ, 408, 428

Owen, F., \& Laing, R. A. 1989, MNRAS, 238, 357

Owen, F., \& Ledlow, M. J. 1994, in The First Stromlo Symposium: The Physics of Active Galaxies, ed. G. V. Bicknell, M. A. Dopita, \& P. J. Quinn (ASP, San Francisco), 319

Owen, F., \& White, R. A. 1991, MNRAS, 249, 164

Owsianik, I., \& Conway, J. E. 1998, A\&A, 337, 69 (OC)

Peletier, R. F., Davies, R. L., Illingworth, G. D., Davis, L. E., \& Cawson, M. 1990, AJ, 100, 1091

Reynolds, C. S., Di Matteo, T., Fabian, A. C., Hwang, U., \& Canizares, C. R. 1996, MNRAS, 283, 111P

Reynolds, C. S., Fabian, A. C., Celotti, A., \& Rees, M. J. 1996, MNRAS, 283, 873

Saikia, D. J., Shastri, P., Cornwell, T. J., \& Banhatti, D. G. 1983, MNRAS, 203, 53P

Sarazin, C. L. 1986, Rev. Mod. Phys., 58, 1

Saripalli, L., \& Gopal-Krishna 1985, A\&A, 149, 205

Scheuer, P. A. G. 1982, in IAU Symp. 97, Extragalactic Radio Sources, ed. D. S. Heeschen, \& C. M. Wade (Kluwer, Dordrecht), 163

Swarup, G., Sinha, R. P., \& Saikia, D. J. 1982, MNRAS, 201, 393

Terlevich, R., Davies, R. L., Faber, S. M., \& Burstein, D. 1981, MNRAS, 196, 381

Valtonen, M. J., \& Heinämäki, P. 2000, ApJ, 530, 107

Wang, Z., Wiita, P. J., \& Hooda, J. S. 2000, ApJ, 534, 201

Wiita, P. J., Rosen, A., \& Norman, M. L. 1990, ApJ, 350, 545

Wiita, P. J., \& Norman, M. L. 1992, ApJ, 385, 478

Zhang, H.-M., Koide, S., \& Sakai, J.-I. 1999, PASJ, 51, 449

Zirbel, E. L. 1997, ApJ, 476, 489 